\title{
Automatic Recognition of Parkinson's Disease Using Surface Electromyography During Standardized Gait Tests
}

\author{
Patrick Kugler ${ }^{1}$, Student Member, IEEE, EMBS, \\ Christian Jaremenko ${ }^{1}$, Johannes Schlachetzki ${ }^{2}$, Juergen Winkler ${ }^{2}$, Jochen Klucken ${ }^{2}$, \\ Bjoern Eskofier ${ }^{1}$, Member, IEEE, EMBS
}

\begin{abstract}
Diagnosis and severity staging of Parkinsons disease (PD) relies mainly on subjective clinical examination. To better monitor disease progression and therapy success in PD patients, new objective and rater independent parameters are required. Surface electromyography (EMG) during dynamic movements is one possible modality. However, EMG signals are often difficult to understand and interpret clinically. In this study pattern recognition was applied to find suitable parameters to differentiate PD patients from healthy controls.

EMG signals were recorded from 5 patients with PD and 5 younger healthy controls, while performing a series of standardized gait tests. Wireless surface electrodes were placed bilaterally on tibialis anterior and gastrocnemius medialis and lateralis. Accelerometers were positioned on both heels and used for step segmentation. Statistical and frequency features were extracted and used to train a Support Vector Machine classifier.

Sensitivity and specificity were high at 0.90 using leave-onesubject-out cross-validation. Feature selection revealed kurtosis and mean frequency as best features, with a significant difference in kurtosis $(p=0.013)$. Evaluated on a bigger population, this could lead to objective diagnostic and staging tools for PD.
\end{abstract}

\section{INTRODUCTION}

Parkinson's disease (PD) is usually diagnosed and clinically characterized by the four major motor symptoms bradykinesia, tremor, rigidity, and postural instability [1]. Assessment of these motor symptoms is performed according to the Unified Parkinson Disease Rating Scale (UPDRS) Part III [2]. This scale defines a series of standardized motor tests to be performed by the patient, which are rated by the clinician according to guidelines. Although video-based teaching programs aim to improve objectivity, evaluation is still subjective and a considerable inter-rater variability has been reported [3]. This limits the comparability of individual assessments and validation of medical interventions.

To resolve these problems and to create an objective assessment of motor impairments different solutions have been proposed. A standard approach is to determine objective gait parameters like step length or foot clearance using markerbased gait analysis [4]. However, this is only performed in specialized gait laboratories and requires complicated setup.

This work was supported by the Embedded Systems Institute (ESI) Erlangen, the Bavarian Ministry for Economic Affairs, Infrastructure, Transport and Technology and the European Fund for Regional Development.

${ }^{1}$ P. Kugler, C. Jaremenko and B. Eskofier are with the Digital Sports Group, Pattern Recognition Lab, Computer Science Department, FriedrichAlexander University of Erlangen-Nuremberg, Erlangen, Germany.

${ }^{2}$ J. Schlachetzki, J. Winkler and J. Klucken are with the Department of Molecular Neurology, University Hospital Erlangen, Erlangen, Germany.

Corresponding author: P. Kugler (patrick.kuglerecs. fau. de)
A recent approach to overcome these drawbacks is to analyze parameters derived from wearable sensors [5]. This includes the analysis of gait patterns [6], [7], foot-pressure [8] or hand motion [9]. Applications are automatic quantification of motor symptoms [5] and monitoring of motor fluctuations [10]. However, these studies focus only on the various motor symptoms resulting from PD and ignore their neromuscular cause, which is a characteristic disturbance in the myoelectric activation of the involved muscles.

Muscle activation is clinically measured using surface electromyography (EMG), which is also used in PD for tremor analysis [11], [12]. Recently, surface EMG signals were also used to discriminate between PD and healthy controls [13], [14]. However, these studies only consider isotonic contractions of the upper extremities, which can only be performed in a clinical setting. Previous work on dynamic contractions includes fatigue during running [15], freezing of gait [16] and signal decomposition [17]. However, none of these studies provides EMG based biomarkers to discriminate PD patients from healthy controls.

The purpose of this study was to automatically classify between PD patients and healthy controls using surface EMG signals from standardized gait tests. The main contributions of this work are as follows: Firstly, a reliable and easy to use study protocol with standardized gait tests was created and applied to collect data from 10 subjects. Secondly, an automated processing pipeline to perform step segmentation, feature extraction and classifier training was set up. Thirdly, the collected data was used to determine expected classification rate and to reveal which muscles, tests and features contribute most to classification accuracy.

\section{METHODS}

\section{A. Study Participants}

In total 10 subjects participated in this study: 5 healthy controls and 5 subjects with Parkinsons disease (PD) according to the consensus criteria of the German Society of Neurology analogue to the National Institute of Neurological Disorders and Stroke (NINDS) diagnostic criteria for PD. Tab. I shows the patient characteristics. The study was approved by the ethics committee (Re.-No. 4208, 2010-21-04, IRB, Medical Faculty, FAU Erlangen-Nuremberg, Germany) and all participating subjects gave written informed consent. 
TABLE I

CHARACTERISTICS OF PATIENTS AND CONTROLS

\begin{tabular}{lcc} 
Characteristic & $\begin{array}{c}\text { PD Patients } \\
(\mathbf{n = 5})\end{array}$ & $\begin{array}{c}\text { Controls } \\
(\mathbf{n}=\mathbf{5})\end{array}$ \\
\hline Sex (male:female) $*$ & $3: 2$ & $4: 1$ \\
Age (y, mean \pm SD) $*$ & $57.0 \pm 16.3$ & $27.6 \pm 1.2$ \\
H\&Y (mean \pm SD) & $1.8 \pm 0.6$ & - \\
UPDRS motorscore (mean \pm SD) & $12.4 \pm 7.6$ & - \\
\hline
\end{tabular}

* Significant difference in gender and age

\section{B. Measurement System}

EMG measurements were performed using Delsys Trigno (Delsys Inc., Boston, MA). This system uses small wireless EMG sensors with four silver bar electrodes and integrated amplifier. The reusable sensors are directly attached to the skin using double sided adhesive tape. This allows fast, simple and hassle-free electrode placement, as no electrode gel or wet electrodes are required. The integrated electrodes reduce artifacts, which is especially important during dynamic movements like gait. Each sensor can also be used as a 3-axis accelerometer, allowing synchronized recording of EMG signals and inertial sensor data.

\section{Electrode Placement}

Trigno EMG electrodes were placed bilaterally on the tibialis anterior (TA), gastrocnemius medialis (GM) and gastrocnemius lateralis (GL) muscles according to SENIAM guidelines. Skin was prepared using abrasive gel and cleaned using isopropyl alcohol to lower skin impedance. Additionally a Trigno Sensor was placed at each heel of the subject to record accelerometer (ACC) data. In total 12 channels were recorded: EMG-TA left/right, EMG-GM left/right, EMGGL left/right and ACC-X/Y/Z left/right. Recording was performed with the Delsys EMGworks software using $4000 \mathrm{~Hz}$ for the EMG channels and $296.3 \mathrm{~Hz}$ for the ACC channels.

\section{Gait Tests}

The Delsys EMGworks software was used to create the measurement protocol, including a signal check and an automatic labeling of all exercises. This allowed reliable recording conditions throughout the study. The measurement protocol consisted of a series of standardized gait tests, which in part correspond to UPDRS Part III [2] and are similar to tests used in previous studies [5], [6]:

- 10-meter walk: The subject walked $10 \mathrm{~m}$ four times at a comfortable walking speed.

- Heel-toe tapping: While the subject was sitting, heel and toes were tapped alternately on the floor for $30 \mathrm{~s}$.

- Foot rotation: While the subject was sitting, a circling movement using only the ankle joint was performed 5$10 \mathrm{~cm}$ above the floor for $30 \mathrm{~s}$.

- Seesaw: While the subject was standing and holding to a handrail, plantar flexion (tiptoe) and dorsiflexion of both foots were performed alternately for $30 \mathrm{~s}$.

\section{E. Filtering and Preprocessing}

The collected data was detrended and filtered according to SENIAM guidelines. EMG signals were filtered between $10 \mathrm{~Hz}$ and $500 \mathrm{~Hz}$ using 4th-order Butterworth filters. The ACC data was low-pass filtered at $2 \mathrm{~Hz}$ using a 4th-order Butterworth low pass filter. To allow easier processing, the filtered ACC data was upsampled to the same sampling rate as the EMG signals, i.e. $4000 \mathrm{~Hz}$.

\section{F. Automatic Step Segmentation}

The recorded data was automatically split into single tests by the recording software. All tests except the 10-meter walk had a defined recording time of $30 \mathrm{~s}$. From these only the middle $15 \mathrm{~s}$ were extracted for further processing. This was done to exclude possible artifacts at the start and end of the exercise and to avoid negative effects due to fatigue.

The data from the 10-meter walk test was additionally processed to automatically segment the continuous signal into single steps. From this only the middle 10 steps of each repetition were used for further processing. This was done to have comparable input data for each subject, as this exercise was performed at an individual walking speed.

Automatic step segmentation was performed separately for each leg using the vertical ACC signal (ACC-Z). The root-mean-square (RMS) energy of each ACC-Z signal was computed over a sliding window of $125 \mathrm{~ms}$ width. The approximate time of heel contact was then extracted by applying peak detection using an empirically determined threshold. Finally, the EMG signals of the corresponding leg were segmented around the time of heel strike using a fixed window of $\pm 300 \mathrm{~ms}$.

After the segmentation step, each subject provided EMG signals for a total of 40 steps á $600 \mathrm{~ms}$ from the 10-meter walk and three sequences á $15 \mathrm{~s}$ from the other three tests.

\section{G. Feature Extraction}

All individual EMG signals from the previous step were used to extract the following statistical features:

- $F_{S 1}$ : Variance

- $F_{S 2}$ : Skewness

- $F_{S 3}$ : Kurtosis

- $F_{S 4}$ : RMS Energy

Additionally, the following frequency features were used:

- $F_{F 1}$ : Dominant Frequency

- $F_{F 2}$ : Mean Frequency

- $F_{F 3}$ : Median Frequency

- $F_{F 4}$ : Total Power

For the 10-meter walk all steps of a single subject were used to compute mean and standard deviation for each feature, leading to 16 features for each muscle in this test. Features from the other three tests were used directly, resulting in only 8 features for each muscle in those tests. In summary $2 * 3 *(16+8+8+8)$ features were extracted, leading to a total of 240 features per subject. 


\section{H. Feature Selection and Ranking}

After the complete feature set was extracted, a forward feature selection was performed [18]. This was done to reduce the number of features and to evaluate which features contributed most to classification accuracy.

The feature selection started out with an empty feature set. In each step one additional feature was added and the classifier was cross-validated on the new feature set. Only the best feature was retained in each step and the process was repeated until cross-validation accuracy did not improve.

The feature selection itself was performed in an additional cross-validation loop to make it robust and to avoid overfitting [19]. This did not lead to a single best feature set, but to multiple feature sets. Feature ranking was performed by counting how often a feature appeared in each feature set.

\section{Classification}

The resulting feature sets were combined into a feature vector and used to train a Support Vector Machine (SVM) classifier [18]. To avoid overfitting due to the small sample size, only a linear kernel was used and the cost parameter $\mathrm{C}$ was fixed to 1. Classification rate was estimated using the leave-one-subject-out cross-validation method. Results were used to calculate average sensitivity and specificity.

\section{EVALUATION}

\section{A. Data Quality}

Data quality of the recording setup was assessed by computing the overall signal-to-noise-ratio (SNR), leading to a SNR of $75.3 \mathrm{~dB}$. Figure 1 shows an example EMG signal collected from a patient with PD. Signal from the GM and GL muscles was generally weaker than from the TA.

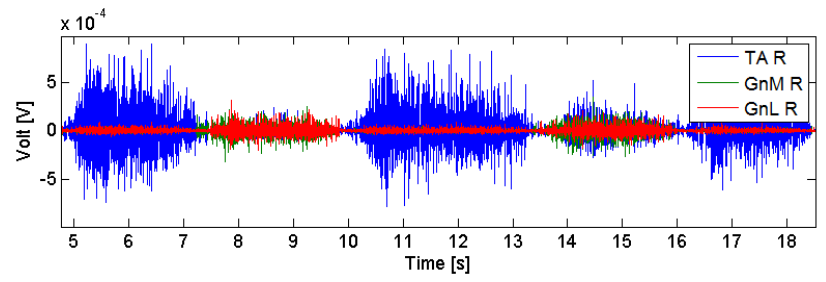

Fig. 1. Example EMG signal of the right tibialis anterior (TA, blue), gastrocnemius medialis (GM, green) and gastrocnemius lateralis (GL, red) during walking.

\section{B. Usability}

To assess usability, subjects were asked about their comfort during recording. They reported that the small wireless sensors were not restricting their motion or interfering with their gait. Testers were asked about the practical usability of the recording setup in a clinical environment. They reported that the system was easy to use with a total time of around 20-30 minutes per patient, including a setup time of 10-15 minutes. Electrode placement was much easier compared to previously used wet electrodes and cables. The implementation of the whole measurement protocol in the recording software decreased time required for a single recording.
Additionally, this increased compliance to the study protocol and no incomplete datasets were recorded.

\section{Automatic Step Segmentation}

The simple step segmentation algorithm was evaluated by visual inspection of the detected steps. A total number of 1233 steps were detected correctly during all recordings. 14 steps were not detected and 9 steps were detected while no or only a partial step was present. Errors were mostly made during turning (missed steps) or at the beginning or end of the test (additional or incomplete steps). Overall the step segmentation algorithm had a sensitivity of $98.9 \%$ and a specificity of $99.3 \%$ when applied to our walking data.

\section{Classification Accuracy}

Classification accuracy was assed using the SVM classifier after feature selection. Experiments were performed (1) using only data from specific muscles, (2) using only data from specific exercises and (3) on the whole data set. Table II shows the results for each experiment.

TABLE II

ClassificATION RESUlts FOR PD vs. CONTROL

\begin{tabular}{lccc} 
Experiment & Sensitivity & Specificity & Best Feature \\
\hline only GM muscle & 0.8 & 1.0 & Mean Freq. GM \\
only GL muscle & 0.8 & 0.8 & Mean Freq. GL \\
only TA muscle & 1.0 & 0.8 & Kurtosis TA \\
\hline only 10-meter walk & 0.8 & 0.6 & Kurtosis TA \\
only Heel-toe-tapping & 0.8 & 0.6 & Mean Freq. TA \\
only Foot rotation & 1.0 & 0.8 & Kurtosis TA \\
only Seesaw & 0.8 & 0.8 & Mean Freq. TA \\
\hline All data & 0.9 & 0.9 & Kurtosis TA \\
\hline
\end{tabular}

\section{E. Feature Ranking}

The best ranking features during feature selection were kurtosis and mean frequency of the TA muscle (Fig. 2), with the difference in kurtosis beeing significant $(p=0.013)$.
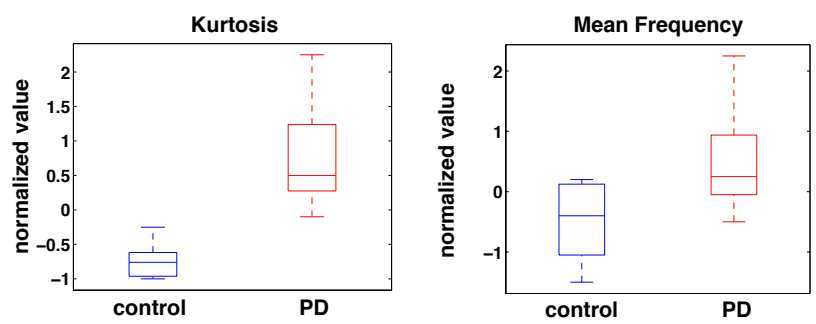

Fig. 2. Comparison of the two best ranked features kurtosis and mean frequency. The difference in kurtosis was significant $(\mathrm{p}=0.013)$.

\section{DISCUSSION}

EMG during dynamic movements like gait is still barely studied in PD. Main reasons for this are (1) the often complicated data collection and (2) the lack of sophisticated analysis algorithms to understand and interpret the signals.

The first important goal of this study was to setup an easy to use recording system to reliably collect high quality EMG data in a clinical environment. This was achieved by 
combining small wireless EMG sensors with a study protocol that includes standardized gait tests. Evaluation showed that the recorded EMG data had a high signal-to-noise-ratio and that the electrodes were non-hindering for the subjects. This allows collecting data during standard assessment in outpatient centers or small clinics.

The second major goal of this work was to determine whether the collected data is usable to build an automatic classification system to discriminate between PD and controls. For this an automated processing pipeline for step segmentation, feature extraction and classification was build.

The evaluation of the step segmentation algorithm showed that it can extract almost all steps correctly. Features were not dependent on an exact time of heel strike, so a rough determination proved sufficient. Missed steps occurred only during turning, however this did not cause a problem as only straight steps were needed. Additional steps at the start or end of an exercise posed no problem, as only the middle steps were needed. Overall the step segmentation proved to be very reliable for the 10-meter walk exercise.

Feature extraction was performed using statistical and frequency features on the unnormalized EMG data of each test or step. Results comparing different muscles indicate that one muscle alone can already provide sufficient information for classification. This suggests that PD affects all EMG signals of one side in the same manner, but further work is needed to support this. Comparison of the different gait tests showed that seesaw and foot rotation had better results than others, but no clear trend could be observed. So far, it is also not clear if step-wise feature extraction improves results. Further analysis with biomechanical gait parameters or time-frequency features like wavelets [15] will clarify this.

Overall classification accuracy was high with sensitivity and specificity at 0.9 when comparing patients with PD to healthy controls. Feature ranking revealed kurtosis as statistically significant feature for the small population, which is consistent with previous results from upper extremities [13].

While the current results must be verified on on a larger age-matched population, they already show that automatic identification of suitable non-linear statistical features is of high value for the discrimination of PD and healthy controls.

\section{CONCLUSION}

This paper presented a study design and classification algorithm to distinguish PD from healthy controls using EMG signals during standardized gait tests. We were able to show that the study design is valid and that the collected data is usable to perform successful classification of PD and controls. Additionally we showed that automatic feature ranking can reveal statistically significant parameters. Evaluation on a bigger and age-matched population is on-going.

In the future, mobile sensors combined with pattern recognition algorithms will lead to an automatic and objective assessment of myoelectric control during gait and will provide valuable tools for diagnosis and staging tools of PD.

\section{ACKNOWLEDGMENT}

We wish to thank all participants of this study and all who helped during data collection. We especially thank Prof. Matthias Lochmann from the Institute of Sports and Sports Science, Erlangen for providing the EMG recording system. Additional support and shoes used in this study were provided by the adidas AG, Herzogenaurach, Germany.

\section{REFERENCES}

[1] J. Jankovic, "Parkinson's disease: clinical features and diagnosis," $J$ Neurol Neurosur Ps, vol. 79, no. 4, pp. 368-376, 2008.

[2] C. G. Goetz et al., "Movement Disorder Society-sponsored revision of the Unified Parkinson's Disease Rating Scale (MDS-UPDRS): Process, format, and clinimetric testing plan," Movement Disorders, vol. 22, no. 1, pp. 41-47, 2007.

[3] B. Post et al., "Unified Parkinson's disease rating scale motor examination: Are ratings of nurses, residents in neurology, and movement disorders specialists interchangeable?" Movement Disorders, vol. 20, no. 12 , pp. $1577-1584,2005$.

[4] M. Mancini et al., "Postural sway as a marker of progression in Parkinson's disease: A pilot longitudinal study," Gait \& Posture, vol. 36, no. 3, pp. 471-476, 2012.

[5] J. Klucken et al., "Unbiased and Mobile Gait Analysis Detects Motor Impairment in Parkinson's Disease," PLoS ONE, accepted, 2013.

[6] J. Barth et al., "Biometric and mobile gait analysis for early diagnosis and therapy monitoring in Parkinson's disease," in Engineering in Medicine and Biology Society EMBC 2011 Annual International Conference of the IEEE, 2011, pp. 868-871.

[7] S. Patel et al., "Longitudinal monitoring of patients with Parkinson's disease via wearable sensor technology in the home setting," in Engineering in Medicine and Biology Society EMBC 2011 Annual International Conference of the IEEE, 2011, pp. 1552-1555.

[8] J. D. Schaafsma et al., "Gait dynamics in Parkinson's disease: relationship to Parkinsonian features, falls and response to levodopa," Journal of the Neurological Sciences, vol. 212, no. 1-2, pp. 47-53, 2003.

[9] J. Barth et al., "Combined analysis of sensor data from hand and gait motor function improves automatic recognition of Parkinson's disease," in Engineering in Medicine and Biology Society EMBC 2012 Annual International Conference of the IEEE, 2012, pp. 5122-5125.

[10] S. Patel et al., "Monitoring Motor Fluctuations in Patients With Parkinson's Disease Using Wearable Sensors," IEEE Trans Inf Technol Biomed, vol. 13, no. 6, pp. 864-873, 2009.

[11] M. Muthuraman et al., "A new diagnostic test to distinguish tremulous Parkinson's disease from advanced essential tremor," Movement Disorders, vol. 26, no. 8, pp. 1548-1552, 2011.

[12] P. Kugler et al., "Automated classification of Parkinson's disease and Essential Tremor by combining electromyography and accelerometer signals," Basal Ganglia, vol. 3, no. 1, p. 61, 2013.

[13] S. M. Rissanen et al., "Discrimination of EMG and acceleration measurements between patients with Parkinson's disease and healthy persons," in Engineering in Medicine and Biology Society EMBC 2010 Annual International Conference of the IEEE, 2010, pp. 4878-4881.

[14] S. Askari et al., "An EMG-based system for continuous monitoring of clinical efficacy of Parkinson's disease treatments," in Engineering in Medicine and Biology Society EMBC 2010 Annual International Conference of the IEEE, 2010, pp. 98-101.

[15] L. M. Stirling et al., "Classification of muscle activity based on effort level during constant pace running," Journal of Electromyography and Kinesiology, vol. 21, no. 4, pp. 566-571, 2011.

[16] B. Cole et al., "Detecting freezing-of-gait during unscripted and unconstrained activity," in Engineering in Medicine and Biology Society EMBC 2011 Annual International Conference of the IEEE, 2011, pp. $5649-5652$.

[17] T. Thrasher et al., "Classification of rhythmic locomotor patterns in electromyographic signals using fuzzy sets," J NeuroEng Rehabil, vol. 8, no. 1, p. 65, 2011.

[18] S. Theodoridis et al., Pattern Recognition (4th Edition). Academic Press, 2008.

[19] K. Fukunaga et al., "Estimation of Classification Error," IEEE Trans Comput, vol. 20, no. 12, pp. 1521-1527, 1971. 